\title{
Antioxidative effects of flavonoids and their metabolites against hypoxia/reoxygenation-induced oxidative stress in a human first trimester trophoblast cell line
}

Vernon J Ebegboni ${ }^{1}$, John M Dickenson ${ }^{2}$ and Shiva D Sivasubramaniam ${ }^{3 凶}$

1vernon.ebegboni@ntu.ac.uk; ${ }^{2}$ john.dickenson@ntu.ac.uk ${ }^{3 凶}$ shiva.sivasubramaniam@ntu.ac.uk

School of Science and Technology, Nottingham Trent University, Clifton Lane, Nottingham NG11 8NS, UK

\section{Abstract:}

This study aimed to investigate the cytoprotective effects of flavonoids, their metabolites alone or in combination against hypoxia/reoxygenation induced oxidative stress in the transformed human first trimester trophoblast cell line (HTR-8/SVneo).

Oxidative stress was achieved with hypoxia followed by reoxygenation and the following assays were performed: MTT, CellTox ${ }^{\mathrm{TM}}$ Green Cytotoxicity, CellTiter-Glo ${ }^{\circledR}$, NADP/NADPH-Glo ${ }^{\mathrm{TM}}$, ROS-Glo ${ }^{\mathrm{TM}} / \mathrm{H}_{2} \mathrm{O}_{2}$, GSH/GSSG-Glo ${ }^{\mathrm{TM}}$ and Caspase-Glo ${ }^{\circledR}$ 3/7 assays.

HTR-8/SVneo cells, pre-treated for $24 \mathrm{~h}$ with flavonoids or their metabolites were protected significantly from oxidative stress. Flavonoids were associated with ROS modulation, reducing the generation of superoxide/hydrogen peroxide. The activities of caspases $3 / 7$ were also significantly reduced significantly in HTR-8/SVneo cells pre-treated with flavonoids.

This study has shown for the first time that $24 \mathrm{~h}$ pre-treatment with flavonoids, their metabolites alone or in combination, protected against HRinduced oxidative stress in the trophoblast cell line. These data indicate that dietary flavonoids may be beneficial to placental health and invasion during early gestation. 
Keywords: trophoblast, antioxidant, flavonoids, quercetin, hesperidin, Q3G, hesperetin oxidative stress, hypoxia/reoxygenation, HTR-8/SVneo

Abbreviations: HR- hypoxia/reoxygenation; Q3G- Quercetin 3-glucuronide 


\subsection{Introduction:}

Flavonoids are a large group of phenolic plant chemicals with significant antioxidant and chelating properties. They are widely distributed in seeds, fruits, vegetables, bark, leaves and flowers. Although their bioactive potentials have been recognised for some time, data relating to their bioavailability, health effects and metabolic fate has come to light only in the past two decades (Banjarnahor \& Artanti, 2014; Iris Erlund, 2004; Heim, Tagliaferro, \& Bobilya, 2002). In addition to their antioxidant properties, flavonoids have been reported to exhibit multiple biological properties. These include anticancer (prostate, breast, colon and lungs), cardioprotection (mainly athero-protection), antiviral, anti-inflammatory, anti-aging and anti-neurodegenerative effects (against Alzheimer's disease) (Daubney, Bonner, Hargreaves, \& Dickenson, 2015; Procházková, Boušová, \& Wilhelmová, 2011; Q. Zhang, Liu, \& Ruan, 2017; Zhang et al., 2015). The mechanisms behind their beneficiary properties is yet to be fully understood. In recent years, emphasis has increased on their antioxidant properties, particularly their ability to scavenge free radicals. However, flavonoids have also been described to have pro-oxidant properties at high concentrations (D. Procházková et al., 2011). It is conceivable that the pro-oxidant properties of flavonoids may trigger protective stress mechanisms that enable tissues/cells to respond to subsequent oxidative stress insults.

Pregnancy is a condition of increased oxidative stress, due to metabolic changes and low-grade inflammation (Ćebović, Marić, Nikolić, \& Novakov-Mikić, 2013). Dietary flavonoids might help support growing demand for antioxidants during early pregnancy. Early human trophoblast invasion occurs in a hypoxic environment (less than $2 \%$ Oxygen; Jauniaux et al., 2000). During this time, uterine spiral arterioles are blocked by endovascular trophoblast cells (Gude, Roberts, Kalionis, \& King, 2004), resulting in release of vasodilator substances, which eventually change 
the spiral arteries from "low-flow high-resistance" to "high-flow low-resistance" vessels (Harris, 2010). However, this short period of hypoxia is also associated with the generation of ROS (reactive oxygen species such as peroxides, superoxide, hydroxyl radical). As these reactive oxygen species are hazardous to the developing placenta and foetus, there is a tendency to increase demand on natural antioxidant defences, such as superoxide dismutase (SOD) and glutathione reductase (GSH) during pregnancy (Ćebović et al., 2013). In support of this, the production of reactive oxygen species was found to be higher during the human pregnancy-related hypertensive disorder preeclampsia (PE), which is believed to result from poor trophoblast invasion (Cross, Tolba, Rondelli, Xu, \& Abdel-Rahman, 2015). Thus, antioxidant therapy has been used during pregnancies (Ly et al., 2015) to reduce oxidative stress and, thereby, the incidence of PE (Kiondo et al., 2014; Rani \& Naidu, 2016; Rumbold \& Crowther, 2005).

Although claims of effective use of vitamin supplements to prevent PE were dismissed by Rossi \& Mullin, (2011), there is still scope for understanding the effects of other naturally occurring antioxidants in fruits and vegetables that would benefit placental health through dietary intake. Therefore, this study aims to investigate the antioxidant properties of the main dietary flavonoids (quercetin, and hesperidin) and their respective metabolites (quercetin-3-glucoside (Q3G) and hesperetin) against hypoxia/reoxygenation-induced oxidative stress in the transformed human first trimester placental cell line HTR-8/SVneo. Here, we report that flavonoids, their metabolites alone or in combination protected against hypoxia/reoxygenation-induced oxidative stress. These findings suggest that consumption of certain foods containing bioactive compounds (flavonoids) might be beneficial for placental health and invasion. 


\section{Materials and Methods}

\subsection{Materials}

Thiazolyl blue tetrazolium bromide (MTT) and all flavonoids/ metabolites used in this study were purchased from Sigma-Aldrich ${ }^{\circledR}$ (Poole, UK) and their purity was as follows: quercetin ( $\geq 95 \%)$, hesperidin ( $\geq 80 \%)$, hesperetin $(\geq 98 \%)$ and quercetin 3-glucuronide ( $\geq 90 \%$ ). Dimethyle sulfoxide (DMSO) was purchased from Fisher Scientific (Loughborough, UK). CellTox ${ }^{\mathrm{TM}}$ green cytotoxicity, CellTiter-Glo ${ }^{\circledR}$ luminescent cell viability, NADP/NADPH-Glo $^{\mathrm{TM}}$, ROS-Glo ${ }^{\mathrm{TM}}$, GSH/GSSG-Glo ${ }^{\mathrm{TM}}$ assays were purchased from Promega (Southampton, UK). RPMI-1640, trypsin $(0.05 \% \mathrm{w} / \mathrm{v})-$ EDTA $(0.02 \% \mathrm{w} / \mathrm{v})$ solution, penicillin $(100 \mathrm{U} / \mathrm{ml})$ and streptomycin $(100 \mu \mathrm{g} / \mathrm{ml})$ were purchased from Lonza (Castleford, UK). Foetal bovine serum (FBS) was purchased from Gibco ${ }^{\circledR}$ (Loughborough, UK). A modular incubator chamber was purchased from BillupsRothenberg Inc. (San Diego, USA). A gas mixture of $0.2 \% \mathrm{O}_{2}, 5 \% \mathrm{CO}_{2}$ and $94.8 \% \mathrm{~N}_{2}$ was bought from BOC Limited (Nottingham, UK) and certified by $\mathrm{HiQ}^{\circledR}$ (Nottingham, UK). Absorbance, fluorescence and luminescence were measured using a FLUOstar Omega plate reader (BMG LABTECH, Aylesbury, UK).

\subsection{In vitro cell culture}

The transformed first trimester human trophoblast cell line (HTR-8/SVneo) was kindly provided by Dr Charles Graham, University of Kingston, Canada (passages used ranged between 32-59). The cells were cultured in RPMI-1640 with L-glutamine supplemented with 10\% (v/v) Foetal Bovine Serum (FBS), penicillin $(100 \mathrm{U} / \mathrm{ml})$ and streptomycin $(100 \mu \mathrm{g} / \mathrm{ml})$ and maintained in a humidified incubator $\left(95 \%\right.$ air, $5 \% \mathrm{CO}_{2}$ at $\left.37{ }^{\circ} \mathrm{C}\right)$ 
until 80-90\% confluent. Following this, they were passaged using trypsin $(0.05 \%$ w/v $)$ - EDTA $(0.02 \%$ w/v) solution and sub-cultured in a T75 flask. Cell culture media were changed every two days. In all experiments, except where indicated, the same protocol was followed. Flavonoids (quercetin and hesperidin) and metabolites (Q3G and hesperetin) were dissolved in dimethyl sulfoxide (DMSO) to make stock solutions and added to the cell culture medium to a final concentration of $0.1 \%$. The same concentration of DMSO $(0.1 \%)$ alone was added to control cells.

\subsection{Hypoxia/reoxygenation (HR) oxidative stress model}

Hypoxia $\left(0.2 \% \mathrm{O}_{2}\right)$ was achieved by placing cultured cells in a humidified modular incubator chamber (Billups-Rothenberg Inc., San Diego, USA), which was flushed with a gas mixture of $0.2 \% \mathrm{O}_{2}, 5 \% \mathrm{CO}_{2}$ and $94.8 \% \mathrm{~N}_{2}$. An electronic oxygen analyser (Teledyne Analytical Instruments, California, USA) was used to confirm the $\mathrm{O}_{2}$ concentration $(0.2 \%)$ before the chamber was sealed. The cells were incubated at $37{ }^{\circ} \mathrm{C}$ for $2 \mathrm{~h}$ followed by reoxygenation for $6 \mathrm{~h}$ in a humidified incubator (95\% air, 5\% $\mathrm{CO}_{2}$ at $\left.37{ }^{\circ} \mathrm{C}\right)$. Both hypoxic and reoxygenation conditions were carried out in serum- and glucose-free media.

\subsection{Cell viability - MTT assay}

Cellular toxicity arising from HR-induced oxidative stress and the effects of flavonoids or their metabolites were analysed in HTR-8/SVneo cells using the methyl thiazolyl tetrazolium bromide method (Sigma-Aldrich, Poole, UK). Briefly, cells were cultured in a 24-well flat-bottomed tissue culture plate at density of $5 \times 10^{4}$ cells per well and cultured for $24 \mathrm{~h}$ in supplemented RPMI-1640. Subsequently, these cells were incubated for 1 
$\mathrm{h}$ in $0.5 \mathrm{mg} / \mathrm{ml}$ MTT before the medium was discarded and the formazan crystals produced dissolved in $200 \mu 1$ of DMSO. Cell viability was determined by measuring absorbance of the solubilized formazan product at $570 \mathrm{~nm}$.

\subsection{CellTox $^{\mathrm{TM}}$ Green Cytotoxicity Assay}

Changes in membrane integrity, which occurred as a result of cell death, in HTR-8/SVneo cells treated with flavonoids or their metabolites were analysed using CellTox ${ }^{\text {TM }}$ green cytotoxicity assay (Promega, Southampton, UK) following the manufacturer's instructions (technical bulletin number TM375). HTR-8/SVneo cells were cultured in a 96-well black with clear bottom tissue culture plate (Falcon ${ }^{\circledR}$, Fisher Scientific, Loughborough, UK) at a density of $2 \times 10^{4}$ cells per well and incubated for overnight to allow the cells to adhere to the surface before treating with flavonoids or their metabolites for $24 \mathrm{~h}$. CellTox ${ }^{\text {TM }}$ green reagent $(2 \mathrm{X})$ was prepared according to the manufacturer's instructions $(1: 1$ with treatment medium) and added to all experimental wells. The plate was mixed briefly using an orbital shaker (500-700 rpm) and kept at room temperature for $15 \mathrm{~min}$. Fluorescence was measured at $485 \mathrm{~nm}$ (excitation) and $520 \mathrm{~nm}$ (emission) using a FLUOstar Omega plate reader (BMG

LABTECH, Aylesbury, UK).

\subsection{CellTiter-Glo ${ }^{\circledR}$ Luminescent Cell Viability Assay}

Cell proliferation, based on quantitation ATP, was measured in HTR-8/SVneo cells treated with flavonoids or their metabolites for $24 \mathrm{~h}$ prior to HR-induced oxidative stress using CellTiter-Glo ${ }^{\circledR}$ Luminescent Cell Viability Assay (Promega, Southmpton, UK) following the manufacturer’s instructions (technical bulletin number TB288). Briefly, cells were cultured in a 96-well black with clear bottom tissue culture plate (Falcon ${ }^{\circledR}$ ) and 
adjusted to a concentration of $5 \times 10^{4}$ cells per well. Cells were incubated overnight before being treating with flavonoids or their metabolites prior to HR exposure. The CellTiter-Glo ${ }^{\circledR}$ substrate was prepared according to the manufacturer's instructions before being added 1:1 to the appropriate (all experimental) wells. Plates were mixed briefly using an orbital shaker ( $2 \mathrm{~min}$ ) to induce cell lysis and kept at room temperature for 30 min. Luminescence was recorded using a FLUOstar Omega plate reader (BMG LABTECH, Aylesbury, UK).

\subsection{NADP/NADPH levels}

Detection of total oxidized and reduced nicotinamide adenine dinucleotide phosphates (NADP ${ }^{+}$and NADPH) was determined using the NADP/NADPH-Glo ${ }^{\text {TM }}$ Assay (Promega, Southampton, UK) following the manufacturer's instructions (technical bulletin number TM400). Cells were cultured, at a concentration of $2 \times 10^{4}$ cells/well, in a 96-well black with clear bottom tissue culture plate $\left(\right.$ Falcon $\left.{ }^{\circledR}\right)$ and incubated overnight before treating with flavonoids or their metabolites for a further $24 \mathrm{~h}$. NADP/NADPH-Glo ${ }^{\mathrm{TM}}$ detection reagent was prepared according to the manufacturer's guidelines. NADP/NADPH ${ }^{\mathrm{TM}}$. Detection reagent was added to samples $(1: 1)$, mixed and kept at room temperature for 60 min. Luminescence was recorded using a FLUOstar Omega plate reader (BMG LABTECH, Aylesbury, UK).

\subsection{ROS-Glo ${ }^{\mathrm{TM}} \mathrm{H}_{2} \mathrm{O}_{2}$ Assay}

Production of hydrogen peroxide $\left(\mathrm{H}_{2} \mathrm{O}_{2}\right)$ was measured using ROS-Glo ${ }^{\mathrm{TM}}$ assay (Promega, Southampton, UKk). Cells were cultured $\left(2 \times 10^{4}\right.$ cells/well) in a 96-well black with clear bottom tissue culture plate (Falcon ${ }^{\circledR}$ ) as previously described. ROS-Glo ${ }^{\mathrm{TM}} \mathrm{H}_{2} \mathrm{O}_{2}$ detection solution was 
prepared according to the manufacturer's guidelines (technical bulletin number TM391) and added to all experimental wells (1:1). After 20 min at room temperature, luminescence was measure using a FLUOstar Omega plate reader (BMG LABTECH, Aylesbury, UK).

\subsection{GSH/GSSG-Glo ${ }^{\mathrm{TM}}$ Assay}

Detecting and quantification of total glutathione (GSH + GSSG), oxidized glutathione (GSSG) and GSH/GSSG ratios was carried out using GSH/GSSG-Glo ${ }^{\mathrm{TM}}$ assay (Promega, Southampton, UK). Cells were cultured and treated as described previously. After hypoxia/reoxygenation insult, the media were discarded and replaced with either total glutathione lysis reagent or oxidized glutathione lysis reagent and the cells were agitated for $5 \mathrm{~min}$. Both plates were kept at room temperature for $30 \mathrm{~min}$ before luciferin generation reagent was added. The plates were briefly agitated in an orbital shaker and allowed to equilibrate at room temperature for 15 min. Luminescence was measured using a FLUOstar Omega plate reader (BMG LABTECH, Aylesbury, UK) and the GSH/GSSG ratio was calculated according to the manufacturer's guidelines.

\subsection{Caspase-Glo ${ }^{\circledR}$ 3/7 Assay}

Caspase-Glo ${ }^{\circledR}$ 3/7 assay (Promega, Southampton, UK) measures caspase-3 and -7 activities in cultured cells. HTR-8/SVneo cells were cultured and treated as described previously. Caspase-Glo ${ }^{\circledR} 3 / 7$ reagent was added to each well before mixing gently on a plate shaker for 30 sec. The plate was kept at room temperature for 45 min before luminescence was recorded using a FLUOstar Omega plate reader (BMG LABTECH, Aylesbury, $\mathrm{UK})$.

\section{Statistical analysis}


All experiments were performed at least three times. Data are presented as mean \pm SEM (standard error of mean) calculated using GraphPad ${ }^{\circledR}$ Prism-7 Software. Statistical analyses were performed using one-way ANOVA followed by Dunnett's multiple comparisons test to determine any differences between the means. A probability value of $\mathrm{P}<0.05$ was considered to be statistically significant 


\subsection{Results and Discussion}

The aim of this investigation was to establish whether flavonoids or their metabolites, which typically occur in food derived from edible plant are associated with reduced HR-induced oxidative stress in early human first trimester extra villous trophoblasts. The role of oxidative stress in the pathophysiology of numerous diseases has been well documented and HR insult leads to reduced trophoblast invasion, resulting in preeclampsia (Feng et al., 2017; Gathiram \& Moodley, 2016; Hung \& Burton, 2006; Sagrillo-Fagundes, Laurent, Bienvenue-Pariseault, \& Vaillancourt, 2018). In this study, we investigated the cytoprotection associated with dietary flavonoids or their metabolites alone or in combination, during HR-induced oxidative stress, examining cell viability, proliferation, apoptosis, ROS modulation and oxidized glutathione prior to HR-induced oxidative stress.

\subsection{Optimization of non-toxic flavonoid concentrations}

To our knowledge, there are no previous studies using the transformed human first trimester early trophoblast cell line (HTR-8/SVneo) in which the impact of non-toxic concentrations of flavonoids and their metabolites on HR-induced oxidative stress is examined. We selected a range of concentrations (1-40 $\mu \mathrm{M}$ ), based on previous studies carried out on non-trophoblast cell lines (Daubney et al., 2015; PeiMing, HoHsing, ChihWen, WenShu, \& ShuJun, 2012; Yamamoto et al., 2013). MTT and CellTox ${ }^{\mathrm{TM}}$ green cytotoxicity assays were used to determine the non-toxic working concentrations of flavonoids (quercetin, hesperidin) and their metabolites (Q3G, hesperetin). As shown in Figure 1 (A-D), which are based on results for the MTT assay, flavonoid concentrations above $10 \mu \mathrm{M}$ were toxic to the cell line HTR-8/SVneo. These results were also validated using the CellTox ${ }^{\mathrm{TM}}$ green cytotoxicity assay (Figure 1, E-H), which monitors cell membrane integrity, and confirmed that concentrations of above 10 
$\mu \mathrm{M}$ triggered cell death. It should be noted that previous studies used concentrations of flavonoids above typical plasma concentrations, which could not be achieved through daily dietary intake of fruits/fruit products. The concentrations of flavonoids used within this study could be achieved from daily intake of fruits and vegetables (Zhang et al., 2015). However, it is difficult to determine amounts that reach the placenta (Feghali, Venkataramanan, \& Caritis, 2015), mainly due ethical constraints around foetal safety. Our results showed that concentrations of flavonoids (quercetin/hesperidin) and their metabolites (Q3G/hesperetin) above $10 \mu \mathrm{M}$ displayed marked toxicity to human trophoblast cell line (HTR8/SVneo).

Since the concentrations of $3 \mu \mathrm{M}$ for flavonoids and $1 \mu \mathrm{M}$ for their metabolites were non-toxic, their combined effects were studied to mimic the situation in vivo, where both flavonoids and their metabolites would occur in tissues. Referencing a previous study by Manach, Morand, GilIzquierdo, Bouteloup-Demange, \& Rémésy (2003), flavonoids are hydrolysed in the liver after a few hours of ingestion. Further studies have revealed that metabolites, as well as the parent flavonoids, are found in both plasma and urine of human subjects (Erlund, Meririnne, Alfthan, \& Aro, 2001; Procházková et al., 2011). Other studies showed that oral administration of quercetin or hesperidin mainly increased plasma concentrations of their metabolites (e.g. Q3G and hesperetin) (Chan et al., 2014; Ting, Yeh, \& Lien, 2011; Yamamoto et al., 2013). In addition, studies have also found that the amounts of metabolites recovered were significantly less than the amounts of flavonoid ingested (Erlund et al., 2001; Procházková et al., 2011). It is thus important to study the combined effects of individual flavonoids and their metabolites. Combinations of flavonoids at $3 \mu \mathrm{M}$ or their respective metabolites at $1 \mu \mathrm{M}$ (quercetin $+\mathrm{Q} 3 \mathrm{G}$ and hesperidin + hesperetin) showed no toxicity. Therefore, these non-toxic concentrations were selected for subsequent experiments. 


\subsection{Oxidative stress model}

An oxidative stress model was achieved by exposing the HTR-8/SVneo cells to either 2 h hypoxia $\left(0.2 \% \mathrm{O}_{2}\right)$ or $2 \mathrm{~h}$ hypoxia followed by $6 \mathrm{~h}$ of re-oxygenation in a humidified incubator $\left(95 \%\right.$ air $/ 5 \% \mathrm{CO}_{2}$ at $\left.37^{\circ} \mathrm{C}\right)$ in serum- and glucose-free media. Exposure of HTR-8/SVneo cells to hypoxia alone increased cell viability (MTT assay). Similarly, the CellTox ${ }^{\mathrm{TM}}$ green cytotoxicity assay revealed no significant difference in cell death between control and hypoxia treated cells. In contrast, upon re-oxygenation (HR), there was a statistically significant reduction in cell viability (MTT assay) and increased cell death $\left(\right.$ CellTox ${ }^{\mathrm{TM}}$ green cytotoxicity assay). There is a considerable body of evidence indicating that trophoblast cells are accustomed to hypoxia (either 0.2 or $2 \% \mathrm{O}_{2}$ ). Our data were in agreement with these studies, suggesting that in HTR-8/SVneo cells, hypoxia alone triggers an increase in cell viability (MTT assay) and reduced cell death (CellTox ${ }^{\mathrm{TM}}$ green cytotoxicity assay). Oxidative stress was established through re-oxygenation, using a protocol modified from previous studies (Armant et al., 2006; Leach, Kilburn, Petkova, Romero, \& Armant, 2008). Hypoxia ( $2 \mathrm{~h}$ ) followed by reoxygenation (6 h) triggered a robust MTT reduction (MTT assay) and decreased membrane integrity in HTR8/SVneo cells, indicating these conditions provided a suitable cell-based model for HR-induced oxidative stress to study the impact of flavonoid supplementation. Therefore, an oxidative stress model was established with re-oxygenation (HR), as hypoxia alone was not sufficient to induce cell death.

\subsection{Effects of flavonoids and metabolites on hypoxia/reoxygenation}


We sought to determine the effects of flavonoids or their metabolites on HR-induced cell death, intracellular ROS levels, and oxidized glutathione. To this end, HTR-8/SVneo cells were treated with flavonoids or their metabolites, alone or in combination, for 24 h prior to HR. The flavonoids quercetin $(3 \mu \mathrm{M})$ and hesperidin $(3 \mu \mathrm{M})$ were associated with significant protection against MTT reduction (Figure 2A and 3A). Similarly, their respective metabolites, Q3G $(1 \mu \mathrm{M})$ and hesperetin $(1 \mu \mathrm{M})$, were also associated with significant protection against HR-induced MTT reduction

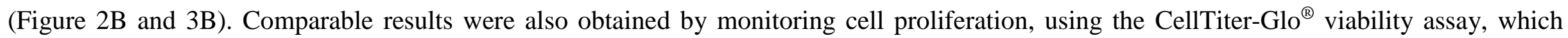
determines intracellular ATP levels (Figure 2D-E and 3D-E). In addition, combinations of flavonoids, with their respective metabolite (quercetin + Q3G and hesperidin + hesperetin), were also associated with statistically significant protection against HR-induced MTT reduction (Figure 2C and 3C) and intracellular ATP levels (Figure 2F and 3F). This data is in agreement with other studies conducted in non-trophoblast cells. Previous studies showed that the presence of quercetin and Q3G was associated with cardioprotection in mitotic and differentiated H9c2 cardiomyocytes (Daubney et al., 2015). Other studies by Ho \& Chang (2004) and Shokoohinia, Hosseinzadeh, Moieni-Arya, Mostafaie, \& Mohammadi-Motlagh (2014) showed that neuronal cells (PC12) pre-incubated with quercetin and Q3G were also protected against $\mathrm{H}_{2} \mathrm{O}_{2}$-induced oxidative stress.

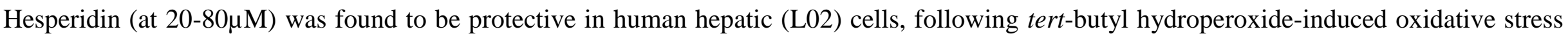
(Chen et al., 2010; Choi \& Ahn, 2008). These authors also reported that hesperidin (at 20-80 $\mu \mathrm{M}$ ) activated antioxidant defence systems in mice subjected to oxidative stress. Indeed, studies have reported that either flavonoids or their metabolites, alone or in combination, could induce antioxidant defence systems (Chen et al., 2010; Shokoohinia et al., 2014). Most importantly, the concentrations used herein fall within a physiologically achievable range. We also investigated the effects of combinations of flavonoid metabolites (quercetin + Q3G and hesperidin + 
hesperetin). Our findings showed this combination was protective against HR-induced oxidative stress. To our knowledge, this is the first report of flavonoid/metabolite combinations being used to protect these cells. Although comparable data from previous studies using trophoblast cells are not available, studies using a pancreatic ductal ademonocarcinoma cell line (BxPc-3) and primary cells showed that combination of flavonoids provide? more protection than single flavonoid treatment during oxidative stress (Appari, Babu, Kaczorowski, Gross, \& Herr, 2014).

\subsection{Effects of flavonoids, metabolites and combinations on ROS}

We examined the effects of flavonoids or their metabolites, alone or in combination, on ROS in HTR8/SVneo cells. As shown in Figures 4 and 5, pre-treatment $(24 \mathrm{~h})$ of cells with quercetin and hesperidin prior to HR was associated with a reduction in superoxide (NADP/NADPH; Figure 4A$\mathrm{C}$ and 5A-C), hydrogen peroxide $\left(\mathrm{H}_{2} \mathrm{O}_{2}\right.$; Figure 4D-F and 5D-F) and oxidized glutathione (GSSG; Figure 4G-I and 5G-1) compared with control cells. Similar results were also obtained for their respective metabolites, Q3G and hesperetin, as well as combinations of flavonoids with their metabolites. Our findings are in agreement with previous studies on non-trophoblast tissues/ cells (Hwang \& Yen, 2011; Jiang, Guo, \& Dusting, 2008; Shaban, Ahmed, El-rashidy, \& Kodous, 2017). For example, in vascular smooth muscle cells, quercetin and its metabolite Q3G inhibited NADPH oxidase and decreased $\mathrm{H}_{2} \mathrm{O}_{2}$ generation (Jiang et al., 2008; Jimenez et al., 2015). The antioxidant properties of flavonoids have been attributed to their ability to scavenge $\mathrm{O}_{2}^{-}$free radical. Indeed, quercetin is a potent scavenger of $\mathrm{O}_{2}^{-}$(Jiang et al., 2008). Another study showed that hesperidin exhibited protection against gamma irradiation in rat testis (Shaban et al., 2017). Furthermore, hesperetin has been found to be neuroprotective against the effects of $\mathrm{H}_{2} \mathrm{O}_{2}$ in PC12 cells (Hwang \& Yen, 2011). Other studies have shown that flavonoids inhibit enzymes responsible for superoxide production (Procházková et al., 2011). Flavonoids have also been shown to activate antioxidants, by inducing phase II 
detoxifying enzymes of the free radical scavenging system (Heim et al., 2002; Procházková et al., 2011). Quercetin and other flavonoids might also protect cells from oxidative stress through metal chelation (Procházková \& Wilhelmová, 2011).

To summarise, our findings showed that HR-induced oxidative stress significantly increased concentrations of GSSG, which is an important bioindicator. These data are similar to another study conducted by Shaban et al. (2017), which showed that gamma radiation-induced oxidative stress increased GSSG concentrations, which decreased following hesperetin treatment. Also, Molina, Sanchez-Reus, Iglesias, \& Benedi (2003) found that, in mouse liver, oxidative stress (triggered by chronic ethanol treatment) induced increased GSSG, which was inhibited by pre-treatment with quercetin and is in agreement with our findings.

\subsection{Effects of flavonoids, metabolites alone or in combinations on caspase $3 / 7$}

We also investigated the effects of flavonoids or their metabolites on apoptosis by assessing caspase 3/7 activities. Both quercetin and hesperidin were associated with a significant reduction in HR-induced caspase 3/7 activation (Figure 6A and 7D). Similarly, Q3G and hesperetin were associated with attenuation of HR-induced caspase 3/7 activation (Figure 6B and 6E). Finally, comparable results were also obtained for flavonoid metabolite combinations (quercetin $+\mathrm{Q} 3 \mathrm{G}$, hesperidin + hesperetin), as shown in Figure $6 \mathrm{C}$ and $6 \mathrm{~F}$. These data suggest flavonoids or their metabolites on apoptosis are protective against HR-induced apoptosis in HTR-8/SVneo cells. Shaban et al. (2017) also found that hesperetin had a protective role against apoptosis induced by gamma radiation in rat testes. Likewise, hesperidin and its metabolite decreased apoptosis (caspase 
3) in neuronal PC12 cells after oxidative stress (Bournival, Quessy, \& Martinoli, 2009). These results were also confirmed by studies in vivo in rats (Ahmad et al., 2012; Shaban et al., 2017; Shagirtha, Bashir, \& Miltonprabu, 2017).

\subsection{Conclusions}

This study has shown for the first time that $24 \mathrm{~h}$ treatment with flavonoids or their metabolites, alone or in combination, is associated with cytoprotective effects against HR-induced oxidative stress in a trophoblast cell line (HTR-8/SVneo). Also, the formation of ROS was reduced significantly by flavonoids or their metabolites in the same trophoblast cell line. The protective effects might be brought about via a reduction in caspase 3/7 activation. Regardless, these findings suggest that increasing intake of fruits and vegetables, rich in flavonoids, might protect against oxidative stress during pregnancy. Further studies are required to explore the complex interplay between the numerous signalling pathways that are modulated by flavonoids and metabolites or their combinations in protecting against oxidative stress in trophoblasts.

\section{Author contributions}

VJE has carried out all the experiments under the supervision of SDS and JMD. Data analysis and manuscript writing were collectively performed by the authors

\section{Acknowledgments}

The work was funded by the Niger Delta Development Commission (NDDC), Nigeria and Dr Ifeanyi Okowa, Governor of Delta State, Nigeria. Special thanks to Michael Shaw, Jacqueline Greef and Gareth Williams for their technical assistance throughout this study. 


\section{Conflict of interest}

The authors declare no conflicts of interest with any other individual researchers or organisations 


\section{References}

Ahmad, S. T., Arjumand, W., Nafees, S., Seth, A., Ali, N., Rashid, S., \& Sultana, S. (2012). Hesperidin alleviates acetaminophen induced toxicity in wistar rats by abrogation of oxidative stress, apoptosis and inflammation. Toxicology Letters, 208(2), 149-161. https://doi.org/10.1016/j.toxlet.2011.10.023

Appari, M., Babu, K. R., Kaczorowski, A., Gross, W., \& Herr, I. (2014). Sulforaphane, quercetin and catechins complement each other in elimination of advanced pancreatic cancer by miR-let-7 induction and K-ras inhibition. International Journal of Oncology, 45(4), 13911400. https://doi.org/10.3892/ijo.2014.2539

Armant, D. R., Kilburn, B. a, Petkova, A., Edwin, S. S., Duniec-Dmuchowski, Z. M., Edwards, H. J., Leach, R. E. (2006). Human trophoblast survival at low oxygen concentrations requires metalloproteinase-mediated shedding of heparin-binding EGF-like growth factor.

Development (Cambridge, England), 133(4), 751-759. https://doi.org/10.1242/dev.02237

Banjarnahor, S. D. S., \& Artanti, N. (2014). Antioxidant properties of flavonoids. Medical Journal of Indonesia, 23(4), 239-244. https://doi.org/10.13181/mji.v23i4.1015

Bournival, J., Quessy, P., \& Martinoli, M. G. (2009). Protective effects of resveratrol and quercetin against MPP+-induced oxidative stress act by modulating markers of apoptotic death in dopaminergic neurons. Cellular and Molecular Neurobiology, 29(8), 1169-1180. https://doi.org/10.1007/s10571-009-9411-5 
Ćebović, T. N., Marić, D., Nikolić, A., \& Novakov-Mikić, A. (2013). Antioxidant Status in Normal Pregnancy and Preeclampsia upon Multivitamin-Mineral Supplementation in the Region of Vojvodina. International Journal of Bioscience, Biochemistry and Bioinformatics, 3(2), 138-144. https://doi.org/10.7763/IJBBB.2013.V3.182

Chan, S. T., Lin, Y. C., Chuang, C. H., Shiau, R. J., Liao, J. W., \& Yeh, S. L. (2014). Oral and intraperitoneal administration of quercetin decreased lymphocyte DNA damage and plasma lipid peroxidation induced by TSA in vivo. BioMed Research International, $2014,580626$. https://doi.org/10.1155/2014/580626

Chen, M., Gu, H., Ye, Y., Lin, B., Sun, L., Deng, W., Liu, J. (2010). Protective effects of hesperidin against oxidative stress of tert-butyl hydroperoxide in human hepatocytes. Food and Chemical Toxicology, 48(10), 2980-2987. https://doi.org/10.1016/j.fct.2010.07.037

Choi, E. J., \& Ahn, W. S. (2008). Neuroprotective effects of chronic hesperetin administration in mice. Archives of Pharmacal Research, 31(11), 1457-1462. https://doi.org/10.1007/s12272-001-2130-1

Cross, C. E., Tolba, M. F., Rondelli, C. M., Xu, M., \& Abdel-Rahman, S. Z. (2015). Oxidative Stress Alters miRNA and Gene Expression Profiles in Villous First Trimester Trophoblasts. BioMed Research International, 2015. https://doi.org/10.1155/2015/257090

Daubney, J., Bonner, P. L., Hargreaves, A. J., \& Dickenson, J. M. (2015). Cardioprotective and cardiotoxic effects of quercetin and two of its in vivo metabolites on differentiated H9c2 cardiomyocytes. Basic and Clinical Pharmacology and Toxicology, 116(2), 96-109. https://doi.org/10.1111/bcpt.12319 
Erlund, I. (2004). Review of the flavonoids quercetin, hesperetin, and naringenin. Dietary sources, bioactivities, bioavailability, and epidemiology. Nutrition Research, 24(10), 851-874. https://doi.org/10.1016/j.nutres.2004.07.005

Erlund, I., Meririnne, E., Alfthan, G., \& Aro, a. (2001). Plasma kinetics and urinary excretion of the flavanones naringenin and hesperetin in humans after ingestion of orange juice and grapefruit juice. The Journal of Nutrition, 131(2), 235-241.

Feghali, M., Venkataramanan, R., \& Caritis, S. (2015). Pharmacokinetics of drugs in pregnancy. In Seminars in Perinatology (Vol. 39, pp. 512519). Elsevier. https://doi.org/10.1053/j.semperi.2015.08.003

Feng, Y., Wang, N., Xu, J., Zou, J., Liang, X., Liu, H., \& Chen, Y. (2017). Alpha-1-antitrypsin functions as a protective factor in preeclampsia through activating Smad2 and inhibitor of DNA binding 4. Oncotarget, 8(68), 113002-113012. https://doi.org/10.18632/oncotarget.22949

Gathiram, P., \& Moodley, J. (2016). Pre-eclampsia: its pathogenesis and pathophysiolgy. Cardiovascular Journal of Africa, 27(2), 71-78. https://doi.org/10.5830/CVJA-2016-009

Gude, N. M., Roberts, C. T., Kalionis, B., \& King, R. G. (2004). Growth and function of the normal human placenta. Thrombosis Research, 114(5-6 SPEC. ISS.), 397-407. https://doi.org/10.1016/j.thromres.2004.06.038

Harris, L. K. (2010). Review: Trophoblast-Vascular Cell Interactions in Early Pregnancy: How to Remodel a Vessel. Placenta, 31(SUPPL.), S93-S98. https://doi.org/10.1016/j.placenta.2009.12.012 
Heim, K. E., Tagliaferro, A. R., \& Bobilya, D. J. (2002). Flavonoid antioxidants: Chemistry, metabolism and structure-activity relationships. Journal of Nutritional Biochemistry, 13(10), 572-584. https://doi.org/10.1016/S0955-2863(02)00208-5

Ho, J. H., \& Chang, Y. L. (2004). Protective effects of quercetin and vitamin C against oxidative stress-induced neurodegeneration. Journal of Agricultural and Food Chemistry, 52(25), 7514-7517. https://doi.org/10.1021/jf049243r

Hung, T.-H., \& Burton, G. J. (2006). 2006-Hypoxia and reoxygenation a possible mechanism for placental oxidative stress in preeclampsia.pdf. Taiwanese Journal of Obstetrics and Gynecology, 45(3), 189-200.

Hwang, S. L., \& Yen, G. C. (2011). Effect of hesperetin against oxidative stress via ER-and TrkA-mediated actions in PC12 cells. Journal of Agricultural and Food Chemistry, 59(10), 5779-5785. https://doi.org/10.1021/jf104632a

Jauniaux, E., Watson, A. L., Hempstock, J., Bao, Y. P., Skepper, J. N., \& Burton, G. J. (2000). Onset of maternal arterial blood flow and placental oxidative stress: A possible factor in human early pregnancy failure. American Journal of Pathology, 157(6), 2111-2122. https://doi.org/10.1016/S0002-9440(10)64849-3

Jiang, F. . b, Guo, N. ., \& Dusting, G. J. . (2008). Modulation of nicotinamide adenine dinucleotide phosphate oxidase expression and function by 3',4'-dihydroxyflavonol in phagocytic and vascular cells. Journal of Pharmacology and Experimental Therapeutics, 324(1), $261-269$. https://doi.org/10.1124/jpet.107.131433

Jimenez, R., Lopez-Sepulveda, R., Romero, M., Toral, M., Cogolludo, a, Perez-Vizcaino, F., \& Duarte, J. (2015). Quercetin and its metabolites 
inhibit the membrane NADPH oxidase activity in vascular smooth muscle cells from normotensive and spontaneously hypertensive rats.

Food \& Function, 6(2), 409-414. https://doi.org/10.1039/c4fo00818a

Kiondo, P., Wamuyu-Maina, G., Wandabwa, J., Bimenya, G. S., Tumwesigye, N. M., \& Okong, P. (2014). The effects of vitamin C supplementation on pre-eclampsia in Mulago Hospital, Kampala, Uganda: A randomized placebo controlled clinical trial. BMC Pregnancy and Childbirth, 14(1), 1-10. https://doi.org/10.1186/1471-2393-14-283

Leach, R. E., Kilburn, B. A., Petkova, A., Romero, R., \& Armant, D. R. (2008). Diminished survival of human cytotrophoblast cells exposed to hypoxia/reoxygenation injury and associated reduction of heparin-binding epidermal growth factor-like growth factor. American Journal of Obstetrics and Gynecology, 198(4), 471. e1-471. e8. https://doi.org/10.1016/j.ajog.2008.01.009

Ly, C., Yockell-Lelièvre, J., Ferraro, Z. M., Arnason, J. T., Ferrier, J., \& Gruslin, A. (2015). The effects of dietary polyphenols on reproductive health and early development. Human Reproduction Update, 21(2), 228-248. https://doi.org/10.1093/humupd/dmu058

Manach, C., Morand, C., Gil-Izquierdo, A., Bouteloup-Demange, C., \& Rémésy, C. (2003). Bioavailability in humans of the flavanones hesperidin and narirutin after the ingestion of two dose of orange juice. European Journal of Clinical Nutrition, 57(2), 235-242. https://doi.org/10.1038/sj.ejen.1601547

Molina, M. F., Sanchez-Reus, I., Iglesias, I., \& Benedi, J. (2003). Quercetin, a flavonoid antioxidant, prevents and protects against ethanolinduced oxidative stress in mouse liver. Biological \& Pharmaceutical Bulletin, 26(10), 1398-1402. https://doi.org/10.1248/bpb.26.1398 
PeiMing, Y., HoHsing, T., ChihWen, P., WenShu, C., \& ShuJun, C. (2012). Dietary flavonoid fisetin targets caspase-3-deficient human breast cancer MCF-7 cells by induction of caspase-7-associated apoptosis and inhibition of autophagy. International Journal of Oncology, 40(2), 469-478. Retrieved from http://www.spandidos-publications.com/ijo/40/2/469

Procházková, D., Boušová, I., \& Wilhelmová, N. (2011). Antioxidant and prooxidant properties of flavonoid. Fitoterapia, 82(4), 513-523. https://doi.org/10.1016/j.fitote.2011.01.01

Procházková, D., \& Wilhelmová, N. (2011). Nitric oxide, reactive nitrogen species and associated enzymes during plant senescence. Nitric Oxide - Biology and Chemistry, 24(2), 61-65. https://doi.org/10.1016/j.niox.2011.01.005

Rani, A., \& Naidu, J. N. (2016). Appraisal of Oxidative Stress Markers, Vitamin E and their Correlation with Blood Pressure in Preeclampsia : A Case Control Study. International Journal of Clinical Biochemistry and Research, 3(1), 11-15. https://doi.org/10.5958/23946377.2016 .00003 .4

Rossi, A. C., \& Mullin, P. M. (2011). Prevention of pre-eclampsia with low-dose aspirin or vitamins C and e in women at high or low risk: A systematic review with meta-analysis. European Journal of Obstetrics Gynecology and Reproductive Biology, 158(1), 9-16. https://doi.org/10.1016/j.ejogrb.2011.04.010

Rumbold, A., \& Crowther, C. A. (2005). Vitamin E supplementation in pregnancy. Cochrane Database of Systematic Reviews. https://doi.org/10.1002/14651858.CD004069.pub2 
S.Ting, Yeh, H. S., \& Lien, T. F. (2011). Effects of supplemental levels of hesperetin and naringenin on egg quality, serum traits and antioxidant activity of laying hens. Animal Feed Science and Technology, 163(1), 59-66. https://doi.org/10.1016/j.anifeedsci.2010.10.001

Sagrillo-Fagundes, L., Laurent, L., Bienvenue-Pariseault, J., \& Vaillancourt, C. (2018). In Vitro Induction of Hypoxia/Reoxygenation on Placental Cells: A Suitable Model for Understanding Placental Diseases. In Preeclampsia (pp. 277-283). Springer.

Shaban, N. Z., Ahmed, A. M., 2^, Z., El-rashidy, F. H., \& Kodous, A. S. A. (2017). Protective role of hesperidin against $\gamma$-radiation-induced oxidative stress and apoptosis in rat testis. Journal of Biological Research-Thessaloniki, 24(245), 1-11. https://doi.org/10.1186/s40709-0170059-x

Shagirtha, K., Bashir, N., \& Miltonprabu, S. (2017). Neuroprotective efficacy of hesperetin against cadmium induced oxidative stress in the brain of rats. Toxicology and Industrial Health, 33(5), 454-468. https://doi.org/10.1177/0748233716665301

Shokoohinia, Y., Hosseinzadeh, L., Moieni-Arya, M., Mostafaie, A., \& Mohammadi-Motlagh, H. R. (2014). Osthole attenuates doxorubicininduced apoptosis in PC12 cells through inhibition of mitochondrial dysfunction and ROS production. BioMed Research International, 2014, 156848. https://doi.org/10.1155/2014/156848

Yamamoto, M., Jokura, H., Hashizume, K., Ominami, H., Shibuya, Y., Suzuki, A., ... Shimotoyodome, A. (2013). Hesperidin metabolite hesperetin-7-O-glucuronide, but not hesperetin-3'-O-glucuronide, exerts hypotensive, vasodilatory, and anti-inflammatory activities. Food \& Function, 4(9), 1346. https://doi.org/10.1039/c3fo60030k 
Zhang, Q., Liu, M., \& Ruan, J. (2017). Metabolomics analysis reveals the metabolic and functional roles of flavonoids in light-sensitive tea leaves. BMC Plant Biology, 17(1), 64. https://doi.org/10.1186/s12870-017-1012-8

Zhang, Y.-J., Gan, R.-Y., Li, S., Zhou, Y., Li, A.-N., Xu, D.-P., \& Li, H.-B. (2015). Antioxidant Phytochemicals for the Prevention and Treatment of Chronic Diseases. Molecules, 20(12), 21138-21156. https://doi.org/10.3390/molecules201219753 


\section{Figure Captions}

\section{Figure 1: Optimization of non-toxic concentrations of flavonoids and their metabolites}

HTR-8/SVneo cells were exposed to the indicated concentrations of flavonoids (quercetin and hesperidin) or metabolites (Q3G and hesperetin) for $24 \mathrm{~h}$. Following flavonoid/metabolite exposure, cell viability was assessed by measuring (A-D) metabolic reduction of MTT by mitochondrial dehydrogenases and $(\mathrm{E}-\mathrm{H})$ membrane integrity via the CellTox ${ }^{\mathrm{TM}}$ green cytotoxicity assay. For the MTT reduction assays the data are expressed as the percentage of control cells $(=100 \%)$ and for CellTox ${ }^{\mathrm{TM}}$ green cytotoxicity assays data are expressed as relative florescence unit. Data represent the mean \pm SEM of $\geq 7$, each performed in quadruplicate. $* P<0.05$ versus control response.

\section{Figure 2: Cytoprotective effects of quercetin, Q3G or in combination}

HTR-8/SVneo cells were treated with quercetin $(3 \mu \mathrm{M})$ or Q3G $(1 \mu \mathrm{M})$ or in combination for 24 h prior to exposure to HR. Cell viability was assessed by measuring (A-C) metabolic reduction of MTT by mitochondrial dehydrogenases and (D-F) intracellular ATP levels using the CelltiterGlo® viability assay. Data are presented as the percentage of control (MTT assay) or relative luminescence units (Celltiter-Glo® viability assay) and represent the mean $+\mathrm{SEM}$ of $\geq 5$, each performed in quadruplicate. ${ }^{*} \mathrm{p}<0.05$ versus control HR.

\section{Figure 3: Cytoprotective effects of hesperidin, hesperetin or in combination.}


HTR-8/SVneo cells were treated with hesperidin $(3 \mu \mathrm{M})$ or hesperetin $(1 \mu \mathrm{M})$ or in combination for $24 \mathrm{~h}$ prior for $24 \mathrm{~h}$ prior to exposure to HR. Cell viability was assessed by measuring (A-C) metabolic reduction of MTT by mitochondrial dehydrogenases and (D-F) intracellular ATP levels using the Celltiter-Glo ${ }^{\circledR}$ viability assay. Data are expressed as the percentage of control (MTT assay) or relative luminescence units (Celltiter-Glo ${ }^{\circledR}$ viability assay) and represent the mean \pm SEM of $\geq 5$ (MTT) or $\geq 4\left(\right.$ CellTiter-Glo $\left.{ }^{\circledR}\right)$ independent experiments each performed in quadruplicate. $*_{\mathrm{p}}<0.05$ versus HR untreated cells.

\section{Figure 4: Scavenging of ROS by quercetin, Q3G or in combination.}

HTR-8/SVneo cells were treated with the indicated concentrations of flavonoids/metabolites or combinations for $24 \mathrm{~h}$ before exposure to HR. ROS production was assessed by (A-C) superoxide generation by measuring NADP/NADPH ratios, (D-F) $\mathrm{H}_{2} \mathrm{O}_{2}$ generation and (G-I) oxidized glutathione (GSSG) generation. Data are presented as relative luminescence units and represent the mean \pm SEM of $\geq 5$ each performed in quadruplicate. ${ }^{*} \mathrm{p}<0.05$ versus HR untreated cells

\section{Figure 5: Scavenging of ROS by hesperidin, hesperetin or in combination.}

HTR-8/SVneo cells were treated with the indicated combinations of flavonoids $(3 \mu \mathrm{M})$ and metabolites $(1 \mu \mathrm{M})$ or in combination for $24 \mathrm{~h}$ prior to exposure to HR. ROS production was assessed by (A-C) superoxide generation by measuring NADP/NADPH ratios, $\left(\right.$ D-F) $\mathrm{H}_{2} \mathrm{O}_{2}$ generation 
and (G-I) oxidized glutathione (GSSG) generation. Data are expressed as relative luminescence units and represent the mean \pm SEM of $\geq 4$ each performed in quadruplicate. ${ }^{*} \mathrm{p}<0.05$ versus HR untreated cells.

\section{Figure 6: The effects of flavonoids, their metabolites alone or in combination on caspase activity}

HTR-8/SVneo cells were treated with flavonoids $(3 \mu \mathrm{M})$ and metabolites $(1 \mu \mathrm{M})$ or in combination for 24 h prior to HR exposure. Following HR, caspase activity was assessed using the Caspase-Glo ${ }^{\circledR} 3 / 7$ assay. Data are presented as relative luminescence units and represent the mean \pm SEM of $\geq 5$ each performed in quadruplicate. $* \mathrm{p}<0.05$ versus HR untreated cells. 

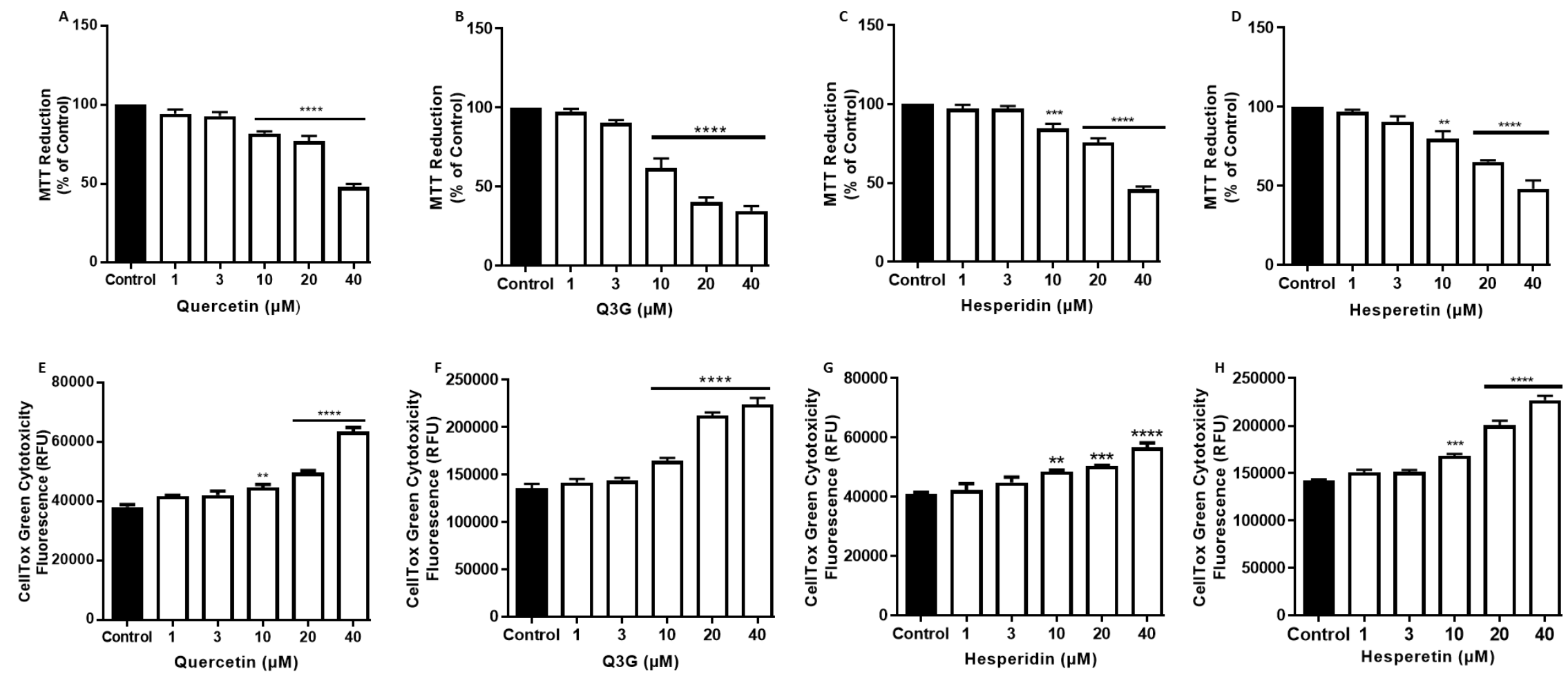

Figure 1: Optimization of non-toxic concentrations of flavonoids and their metabolites 

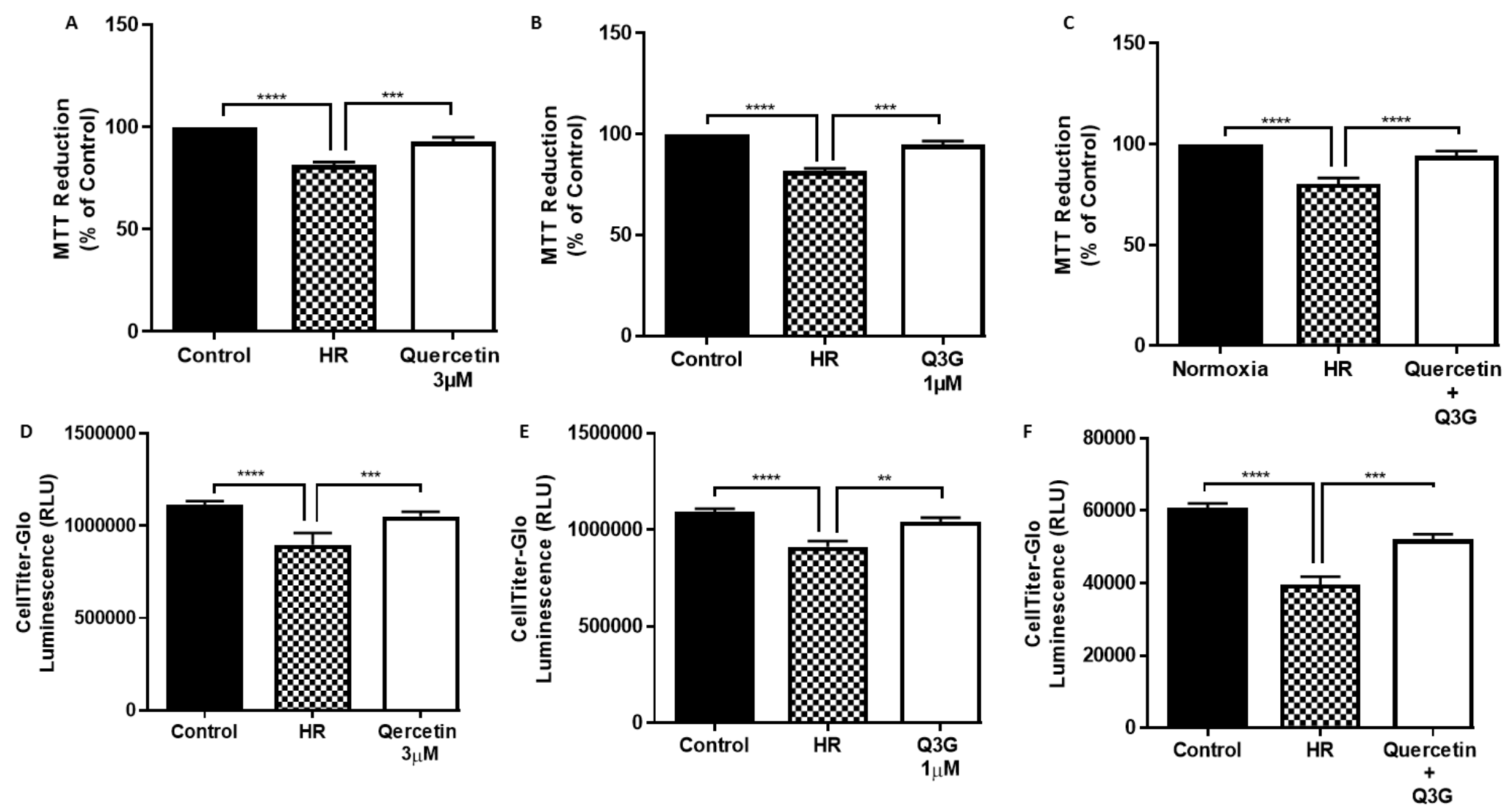

Figure 2: Cytoprotective effects of quercetin, Q3G or in combination 

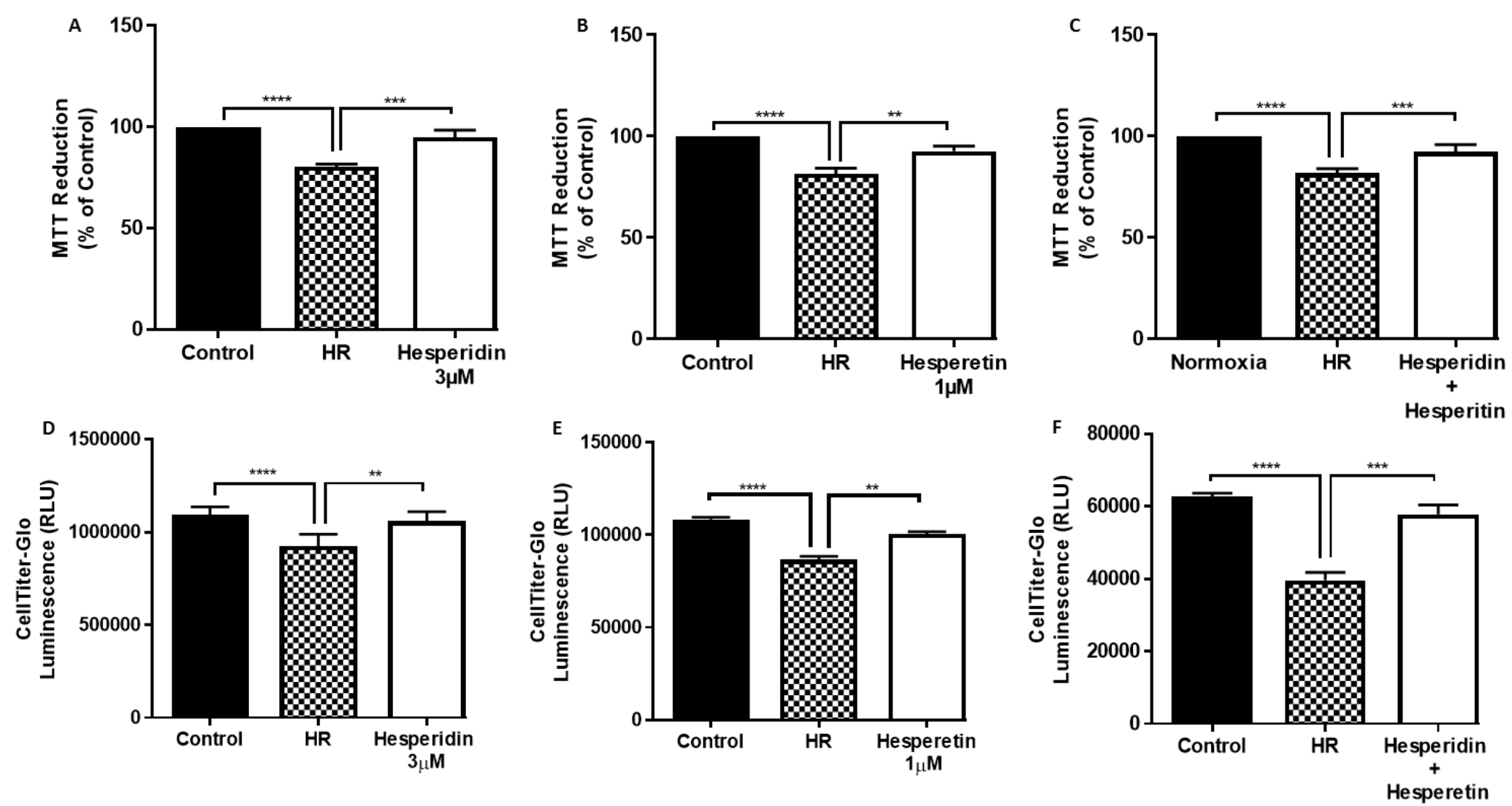

Figure 3: Cytoprotective effectsof hesperidin, hesperetin or in combination. 

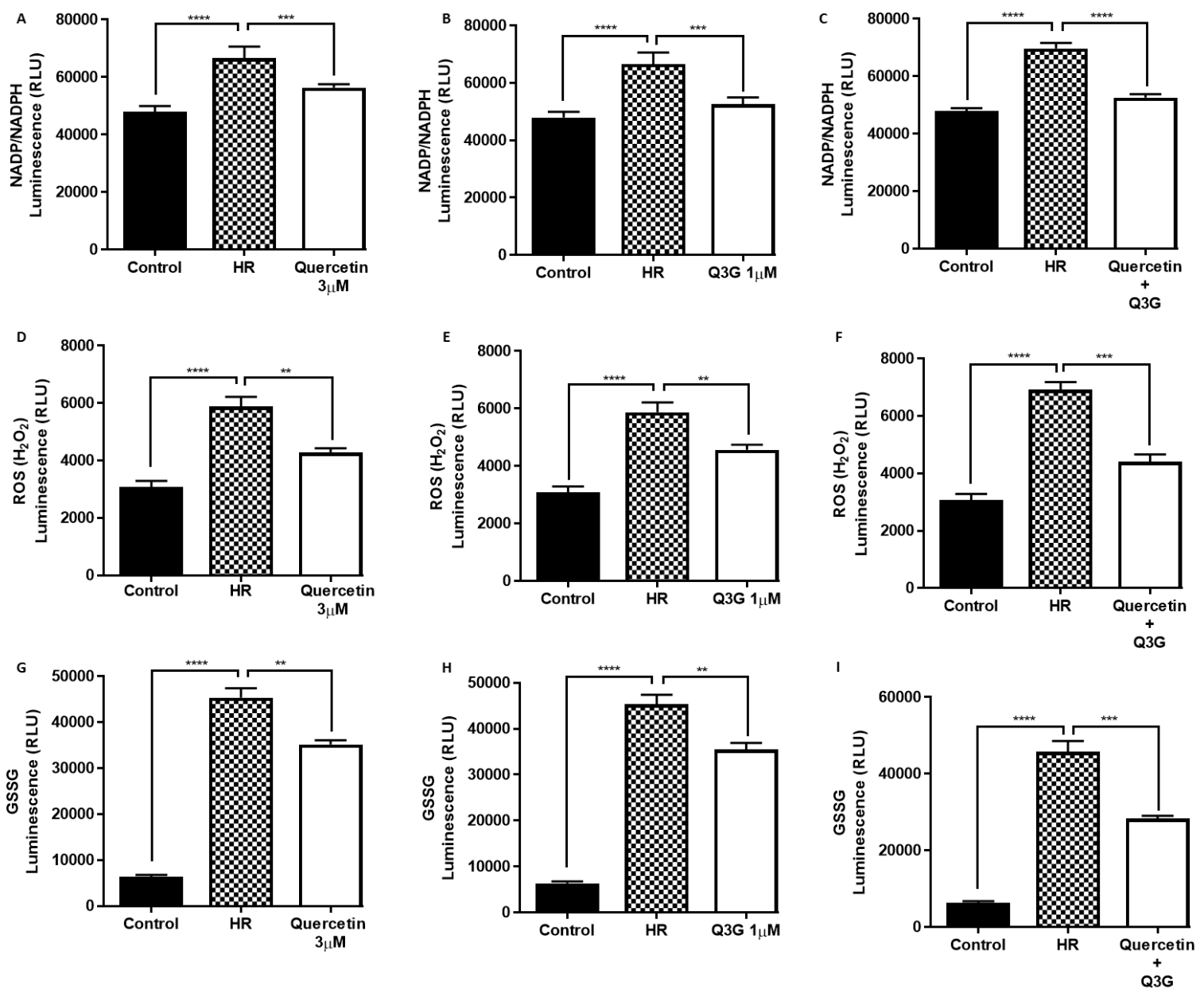

Figure 4: Scavenging of ROS by quercetin, Q3G or in combination. 

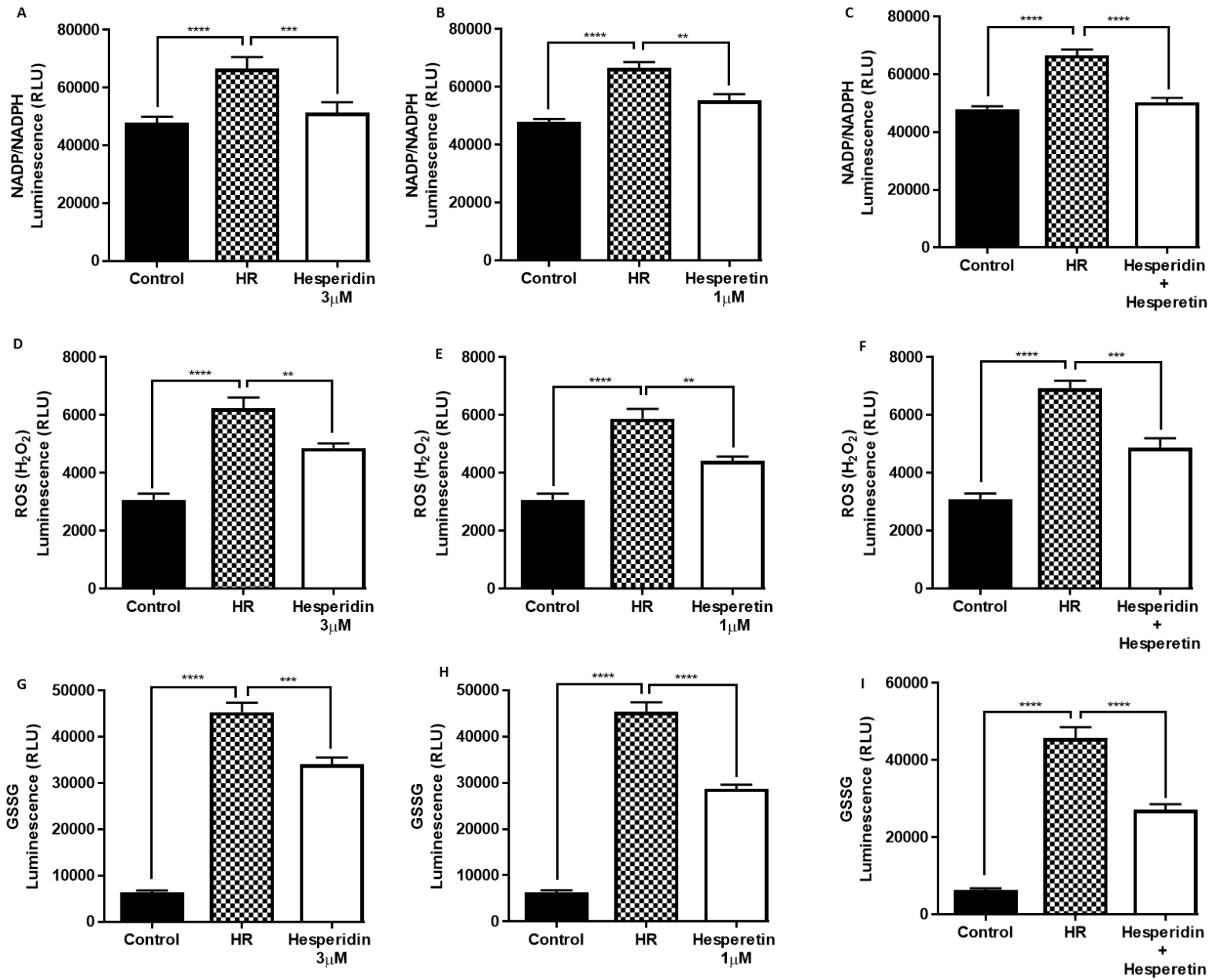

Figure 5: Scavenging of ROS by hesperidin, hesperetin or in combination. 

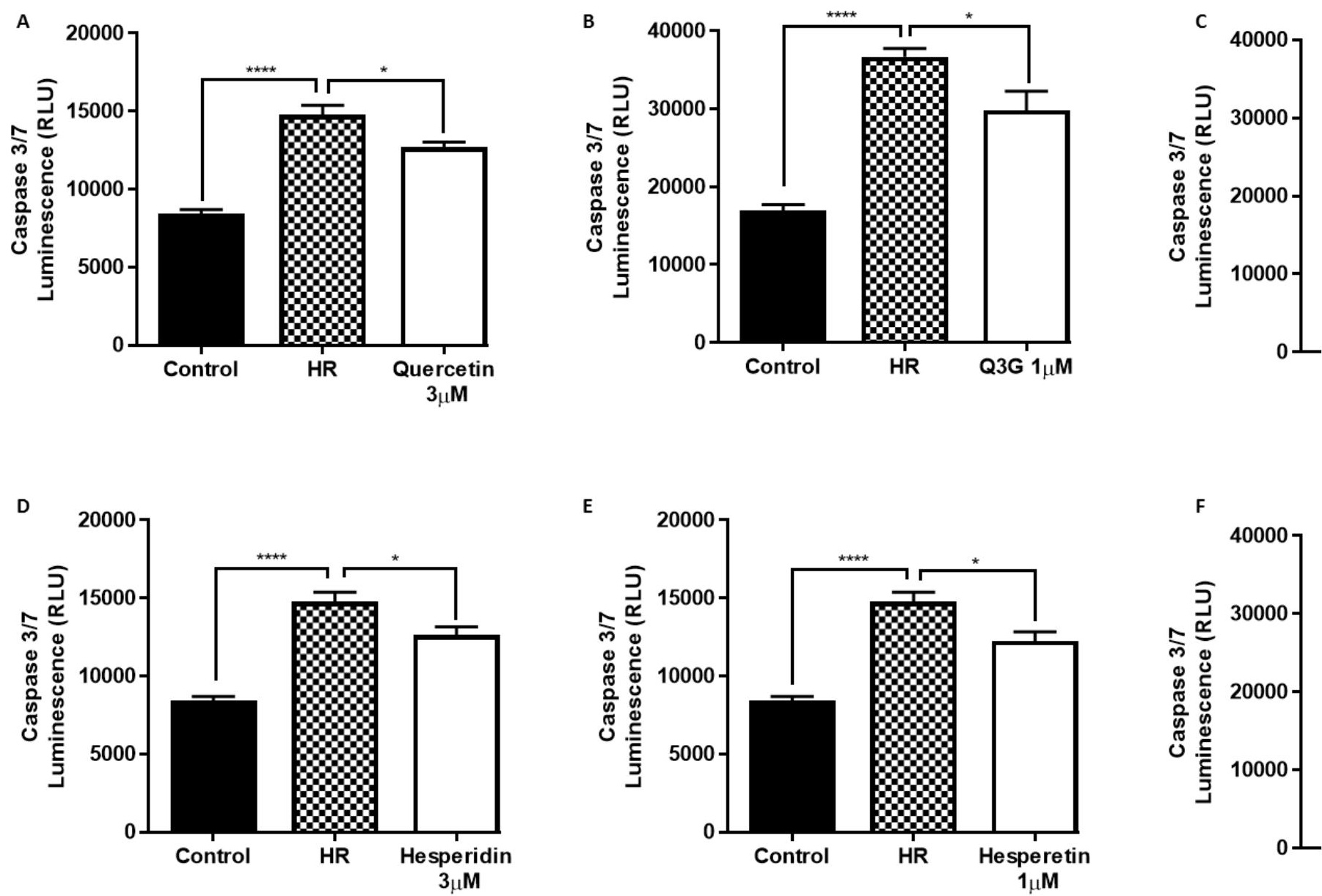

1

2 Figure 6: The effects of flavonoids, their metabolites alone or in combi 